\title{
Atropine penalisation versus occlusion as the primary treatment for amblyopia
}

\author{
Aisling Foley-Nolan, Aoife McCann, Michael O'Keefe
}

\begin{abstract}
Aims/background-Pharmacological penalisation of non-amblyopic eyes is an infrequently used alternative to occlusion for treating amblyopia. The authors compared the efficacy of atropine penalisation and that of occlusion as a primary treatment for amblyopia.

Methods-Thirty six newly diagnosed patients with amblyopia were allocated to two groups for treatment. Eighteen patients in each group were treated either with atropine penalisation (group A) or occlusion therapy (group P).

Results-There was a statistically significant improvement in visual acuity in both groups treated. In group $A$ improvement of the geometric mean visual acuity of the amblyopic eye was from $6 / 50$ to $6 / 11$ $(p<0.001)$. In group $P$ improvement of the geometric mean visual acuity was from $6 / 60$ to $6 / 19(p<0.001)$. In group A noncompliance with treatment was only $6 \%$ (2/18). Non-compliance in group $P$ was $45 \%(8 / 18)$ at some stages of the treatment. Neither group produced an incidence of occlusion amblyopia.

Conclusions-In this study atropine penalisation has been shown to be as effective as occlusion therapy in the treatment of amblyopia. Patient acceptance of atropine penalisation was superior to that for occlusion therapy as was shown by the compliance rate. Atropine treatment was also advantageous in that compliance could be readily checked by inspection.

(Brf Ophthalmol 1997;81:54-57)
\end{abstract}

Occlusion of the non-amblyopic eye is the most commonly used treatment for children with amblyopia. Pharmacological penalisation is an alternative to occlusion in the treatment of this condition. ${ }^{1}$ Pharmacological penalisation involves the daily instillation of a cycloplegic agent into the fornix of the non-amblyopic eye to prevent accommodation, thus causing the patient to prefer the amblyopic eye at near.

Pharmacological penalisation is infrequently used. Repka and Ray reported on the efficacy of optical and pharmacological penalisation in $1993 .^{2}$ They reported an improvement in visual acuity in $76 \%$ of 79 amblyopic eyes treated with pharmacological penalisation. In our study atropine penalisation was compared with occlusion therapy as a first line treatment for patients with significant (all of the patients had initial visual acuity of $6 / 18$ or less) amblyo- pia. This comparison of treatments has not been reported in the literature before.

\section{Materials and methods}

All of the patients entering into this trial were new patients who presented to our outpatient clinic having never had any previous treatment for their amblyopia. The trial began in January 1994, and the last patient was entered in December 1994. All new patients due to commence treatment for amblyopia were allocated either to treatment with atropine penalisation (treatment A), or to occlusion therapy (treatment $\mathrm{P}$ ). This was achieved on a strict alternate patient basis. Appointments were organised by an independent observer (clinic sister) in order to prevent any possibility of bias being introduced.

Data collected included age, previous and family ocular history, type of amblyopia, visual acuity, and refractive error. Visual acuity was determined using the Snellen chart, Kay's pictures, or Sheridan-Gardener test types, depending on the age and comprehension of the patient. At each visit the visual acuity was assessed by two independent observers. Disagreements between the two observers were resolved by averaging the reported visual acuity. The visual acuity assessors were masked to patient treatment. Refractive error was examined by cycloplegic retinoscopy $35 \mathrm{~min}$ utes after instillation of $1 \%$ cyclopentolate. Each of these data elements was determined at the time of the first examination, the conclusion of therapy, and after the longest term follow up.

Treatment was considered to have been concluded when a visual acuity of $6 / 6$ was achieved, or when visual acuity remained static over three successive assessments.

\section{TREATMENT P}

An occlusive patch was placed over the non-amblyopic eye for a predetermined amount of time each day. The protocol for the amount of occlusion varied according to the age of the child, and the amount of amblyopia. We patched full time for 1 week per year of life-for example, 4 weeks of full time patching for a 4-year-old child. Visual acuity was then reassessed at the next visit and if it had recovered to $6 / 9$ or better occlusion was reduced to half days for fear of producing occlusion amblyopia. Patients were monitored weekly per year of life.
Accepted for publication 4 October 1996 
Table 1 Demographic characteristics of treatment groups $A$ and $P$

\begin{tabular}{ll}
\hline Treatment A (n =18): & \\
Mean age & 5.5 years \\
Frequency of strabismus & $95.2 \%(17 / 18)$ \\
Frequency of anisometropia & $88.9 \%(16 / 18)$ \\
Mean spherical error & $+5.5 \mathrm{DS}$ \\
Mean duration therapy (months) & 7.2 \\
Treatment P (n = 18): & 5.5 years \\
Mean age & $88.9 \%(16 / 18)$ \\
Frequency of strabismus & $66.7 \%(12 / 18)$ \\
Frequency of anisometropia & $+4.6 \mathrm{DS}$ \\
Mean spherical error & 4.3 months \\
Mean duration therapy (months) & \\
\hline
\end{tabular}

TREATMENT A

One drop of atropine $1 \%$ was placed in the conjunctival fornix of the non-amblyopic eye each morning.

For each of the treatments an information sheet was given to the parents detailing the treatment, objectives, and complications. Informed consent to entering the trial was obtained from the parents in all cases.

Follow up visits were on an approximate monthly basis, but were also determined by age and degree of patching. Data retrieval at follow up visits consisted of assessment of compliance and tolerance of treatment, in addition to the other measurements already outlined. Parents were asked to monitor compliance closely at home. At each visit parents were asked to rate compliance, poor (treatment used less than a third of time), average (treatment used two thirds of time), good (treatment used all of time). Atropine compliance was assessed by inquiry of parents and also objectively by checking pupil fixation. Side effects were also noted-for example, allergy or photosensitivity.

STATISTICAL ANALYSIS

The geometric mean of each visual acuity was calculated in the manner described by Holladay and Prager. ${ }^{3}$ The logarithm of each visual acuity was calculated. The average of these values was obtained. The means were compared using paired Student's $t$ tests. The antilog of the geometric mean was calculated and the resultant converted to Snellen notation for reporting the mean acuity.

\section{Results}

Thirty six patients were treated with either atropine penalisation or occlusion therapy-18 patients in each group. The distribution of age, frequency of strabismus, and mean spherical correction were balanced between the two treatment groups (Table 1).

The patients in group A treated with atropine penalisation ranged in age from 2.5 to 9 years (mean 5.5 years). The patients in group $\mathrm{P}$ treated with occlusion therapy also had an age range of 2.5 to 9 years with a mean age of 5.5 years. All 36 patients were able to perform Snellen, Sheridan-Gardener, or Kay's pictures tests at the beginning of the treatment. In group A the frequency of strabismus was $95.2 \%$ and that of anisometropia $88.9 \%$. In group $\mathrm{P}$ the frequency of strabismus was $88.9 \%$, and that of anisometropia $66.7 \%$. The
Table 2 Mean visual acuities*

\begin{tabular}{|c|c|c|c|}
\hline & $\begin{array}{l}\text { Before } \\
\text { penalisation }\end{array}$ & $\begin{array}{l}\text { End of } \\
\text { penalisation }\end{array}$ & $p$ Value \\
\hline \multicolumn{4}{|l|}{ Treatment A group: } \\
\hline $\begin{array}{l}\text { Amblyopic } \\
\text { eyes }\end{array}$ & $6 / 50$ & $6 / 11$ & $<0.001$ \\
\hline Penalised eyes & $6 / 7$ & $6 / 7$ & NS \\
\hline \multicolumn{4}{|l|}{ Treatment $\mathrm{P}$ group: } \\
\hline $\begin{array}{l}\text { Amblyopic } \\
\text { eyes }\end{array}$ & $6 / 60$ & $6 / 19$ & $<0.001$ \\
\hline Penalised eyes & $6 / 7$ & $6 / 7$ & $<0.001$ \\
\hline
\end{tabular}

mean spherical correction required for patients in group A was +5.5 dioptre spheres, and that for patients in group $\mathrm{P}$ was +4.6 dioptre spheres. The length of treatment for group A was 1-12 months, with a mean 7.2 months. The length of treatment for group P was 2-9 months with a mean of 4.3 months. Compliance with treatment in the two groups was $94 \%$ for group A and $55 \%$ for group P.

Initial acuities of the amblyopic eye in the 18 patients in group A ranged from $6 / 18$ to $6 / 120$ (see appendix 1) with a geometric mean of $6 / 50$. Initial acuities of the amblyopic eye in the 18 patients in group $P$ ranged from $6 / 18$ to $6 / 120$ (see appendix 2) with a geometric mean of $6 / 60$. At the end of the course of treatment, acuities in group A ranged from $6 / 6$ to $6 / 60$ with a geometric mean of $6 / 11$.

Post treatment acuities in group $\mathrm{P}$ ranged from $6 / 6$ to $6 / 120$ with a geometric mean of $6 / 19$. There was a statistically significant improvement in visual acuity in both groups in the amblyopic eye following treatment: for group A Student's $t$ test, $-5.79 ; \mathrm{df} 17 ; \mathrm{p}<0.001$; for group P Student's $t$ test, -5.46 ; df 17; $\mathrm{p}<0.001$ (see Table 2).

\section{Discussion}

The goal in the treatment of amblyopia is to restore visual acuity in the affected eye and, once this has been accomplished, to prevent recurrence of the disorder. The traditional treatment for amblyopia is occlusion of the dominant eye and forced use of the amblyopic eye. ${ }^{4-6}$ Conventional occlusion therapy is not feasible in all amblyopic children, however, because of skin sensitivity or allergy to adhesive material, latent nystagmus, emotional problems caused by wearing a patch, or total lack of cooperation. ${ }^{78}$ Penalisation, which reduces the visual acuity of the sound eye pharmacologically or optically, may be an alternative treatment for such patients. ${ }^{78}$ The principle of this method was developed by Worth, ${ }^{9}$ who reported recovery of visual acuity of amblyopic eyes after prolonged administration of atropine to the sound eye, an observation since confirmed by other investigators. ${ }^{10-12}$

Atropine penalisation therapy was readily accepted in our study, and there was never any pressure from the patients to stop treatment. In fact there was often pressure to continue, just in case greater success could be achieved. This was in stark contrast with treatment with 
occlusion therapy, where there was constant pressure from both patients and parents to terminate the treatment. In our study mean duration of atropine treatment was 7.2 months as opposed to 4.3 months for the occlusion group.

The reason for this was that we were continuing to get improvement in visual acuity in the atropine group. According to our protocol treatment was to continue until a visual acuity of $6 / 6$ was achieved or, when visual acuity remained static, over three successive visits.

The ability to treat for a longer period during visual development would appear to be a definite advantage of penalisation over occlusion since it is known that deterioration of effect can be something experienced by up to $55 \%$ of patients after occlusion therapy has stopped. ${ }^{13}$

The neurophysiology of amblyopia from single unit recordings in amblyopic macaque monkeys provides insight as to why atropine penalisation is effective. Both anisometropic and strabismic amblyopia show a loss of responses in neurons tuned for high spatial frequencies at the supragranular layers of striate cortex. ${ }^{14}$ What occurs is that high spatial frequency neurons from the non-amblyopic eye are crowding out high spatial frequency neurons from the amblyopic eye. In order to treat the amblyopic eye the high spatial frequency neurons from the dominant eye need to be shut down. The blur produced by atropine has the effect of selectively removing the high spatial frequency components of the image, allowing the low spatial frequency neurons through. In this way we have the effect of stimulating vision in the amblyopic eye without totally disrupting binocularity, which is what occurs in occlusion therapy. There were no cases of occlusion amblyopia in either group of patients in this series. However, we must be wary of its occurrence. North and Kelly ${ }^{15}$ reported two cases in 189 who suffered permanent occlusion amblyopia after pharmacological penalisation. The low incidence of occlusion amblyopia is because the penalised eye is still receiving low frequency information.

A potential disadvantage of penalisation with atropine is the effect that the cycloplegia may have on the development of a myopic refractive error. This is based on experimental work in animals and case reports in humans; however, no refractive changes occurred in the patients involved in this study.

A useful aspect of atropine penalisation over occlusion therapy observed in this study was the ability of the examiner to check compliance. With atropine use there is a fixed dilated pupil, there is no such telltale sign when wearing an occlusive patch. This can be helpful in cases where the patient and/or parent have selective memories! It can readily be seen that the atropine is not being used, and we can reinforce the message of the importance of compliance, in trying to bring about the result that we all want - that is, maximal visual acuity in the shortest amount of treatment time possible.

With prolonged use of topical atropine in children the incidence of contact sensitivity is surprisingly low. ${ }^{16}$ None of the 18 patients using atropine in this study developed irritation of the eyelids. Parents are advised to instil only one drop, and to wipe from the skin any excess that might spill from the eyelid. This careful skin toilet is very beneficial so that skin sensitivity to atropine causes less irritation than was formerly caused by plasters. Atropine toxicity is very rare with suitable preparations appropriately applied. Aqueous atropine eyedrops thickened with methylcellulose, which were used in this study, undergo little nasopharyngeal absorption, and no patient has required the discontinuance of atropine because of toxicity.

This is the first study comparing atropine penalisation with occlusion therapy in the literature. Whereas we accept that there are limitations to a study of this size, we found atropine to be as effective as occlusion and to have a higher degree of patient acceptability. These are important and highly useful findings in what, after all, is the commonest of all problems in paediatric ophthalmology.

1 Repka MX, Gallin PF, Scholz RT, Guyton DL. Determination of optical penalisation by vectographic fixation reversal. Ophthalmology 1985;92:1584-6.

2 Repka MX, Ray JM. The efficacy of optical and pharmacological penalisation. Ophthalmology 1993;100:769-75

3 Holladay JT, Prager TC. Mean visual acuity.[Letter] Am $\mathcal{f}$ Ophthalmol 1991;111:372-4.

4 Von Noorden GK. Factors involved in the production of Von Noorden GK. Factors involved in the pro
amblyopia. Br $\mathcal{F}$ Ophthalmol 1974;58:158-64.

5 Sloper JJ. Edbridge-Green lecture. Competition and cooperation in visual development. Eye 1993;7:319-31.

6 Rogawski MA. New directions in neurotransmitter action: dopamine provides some important clues. TINS 1987;10: 200 .

7 Swann AP, Hunter CD. A survey of amblyopia treated by atropine occlusion. Br Orthopt f 1974;31:65-9.

8 Von Noorden GK, Milam JB. Penalization in the treatment of amblyopia. Am f Ophthalmol 1979;88:511-8.

9 Worth C. Squint: its causes, pathology, and treatment. London: John Bales, Sons and Danielson, 1903:71,73,108.

10 Guibor P. The use of atropine in the treatment of motor defects. In: Allen JH, ed. Strabismus ophthalmic symposium. St Louis: CV Mosby, 1950:316.

11 Lowe RF. The use of atropine in the treatment of amblyopia ex anopsia. Med $\mathcal{F}$ Aust 1963;5:725.

12 Knapp P, Capobianco N. Use of miotics in esotropia. Am Orthopt $\mathcal{F} 1971 ; 28: 77$.

13 Levarotsky S, Gottesman N, Shimshoni N, Oliver M. Factors affecting long-term results of successfully treated amblyopia: age at beginning of treatement and age at cessation of monitoring. $\mathcal{F}$ Pediatr Ophthalmol Strabismus 1992;29:219-23.

14 Movshon JA, Eggers HM, Gizzi MS, Hendrickson AE, Kiorpes L, Boothe RG. Effects of early unilateral blur on the macaque's visual system. 111. Physiological observations. F Neurosci 1987;7:1340-51.

15 North RV, Kelly ME. Atropine occlusion in the treatment of strabismic amblyopia and its effect on the non-amblyopic eye. Ophthalmic Physiol Opt 1991;11:113-7.

16 Lowe RF. Atropine treatment for amblyopia ex anopsia. $\mathrm{Br}$ Orthopt f 1965;22:35-42. 


\begin{tabular}{llll}
$\begin{array}{l}\text { Appendix } 1 \\
\text { amblyopic eye }\end{array}$ & $\begin{array}{l}\text { Visual acuities of treatment A group in the } \\
\text { Before } \\
\text { treatment }\end{array}$ & \multicolumn{4}{l}{$\begin{array}{l}\text { After treatment } \\
\text { treatment }\end{array}$} & After treatment \\
\hline $16 / 120 \mathrm{SN}$ & $6 / 18 \mathrm{SN}$ & $26 / 60 \mathrm{KP}$ & $6 / 6 \mathrm{KP}$ \\
$36 / 36 \mathrm{SN}$ & $6 / 9 \mathrm{SN}$ & $46 / 18 \mathrm{SG}$ & $6 / 6 \mathrm{SG}$ \\
$56 / 36 \mathrm{SN}$ & $6 / 12 \mathrm{SN}$ & $66 / 60 \mathrm{SN}$ & $6 / 9 \mathrm{SN}$ \\
$76 / 60 \mathrm{SN}$ & $6 / 60 \mathrm{SN}$ & $86 / 60 \mathrm{KP}$ & $6 / 24 \mathrm{KP}$ \\
$96 / 120 \mathrm{SN}$ & $6 / 18 \mathrm{SN}$ & $106 / 18 \mathrm{SG}$ & $6 / 9 \mathrm{SG}$ \\
$116 / 120 \mathrm{SG}$ & $6 / 9 \mathrm{SG}$ & $126 / 120 \mathrm{SG}$ & $6 / 6 \mathrm{SG}$ \\
$136 / 18 \mathrm{SN}$ & $6 / 18 \mathrm{SN}$ & $146 / 24 \mathrm{SG}$ & $6 / 6 \mathrm{SG}$ \\
$156 / 60 \mathrm{SG}$ & $6 / 18 \mathrm{SG}$ & $166 / 18 \mathrm{KP}$ & $6 / 9 \mathrm{KP}$ \\
$176 / 120 \mathrm{SG}$ & $6 / 6 \mathrm{SG}$ & $186 / 24 \mathrm{SN}$ & $6 / 6 \mathrm{SN}$ \\
\hline
\end{tabular}

$\mathrm{SN}=$ Snellen, $\mathrm{SG}=$ Sheridan-Gardener, $\mathrm{KP}=$ Kay's pictures .
Appendix 2 Visual acuities of treatment $P$ group in the amblyopic eye

\begin{tabular}{llll}
\hline $\begin{array}{l}\text { Before } \\
\text { treatment }\end{array}$ & After treatment & $\begin{array}{l}\text { Before } \\
\text { treatment }\end{array}$ & After treatment \\
\hline $16 / 60 \mathrm{SN}$ & $6 / 9 \mathrm{SN}$ & $26 / 36 \mathrm{KP}$ & $6 / 6 \mathrm{KP}$ \\
$36 / 60 \mathrm{SN}$ & $6 / 60 \mathrm{SN}$ & $46 / 120 \mathrm{SN}$ & $6 / 18 \mathrm{SN}$ \\
$56 / 60 \mathrm{SN}$ & $6 / 6 \mathrm{SN}$ & $66 / 120 \mathrm{SG}$ & $6 / 24 \mathrm{SG}$ \\
$76 / 60 \mathrm{SN}$ & $6 / 6 \mathrm{SN}$ & $86 / 24 \mathrm{SN}$ & $6 / 6 \mathrm{SN}$ \\
$96 / 120 \mathrm{KP}$ & $6 / 120 \mathrm{KP}$ & $106 / 120 \mathrm{SG}$ & $6 / 120 \mathrm{SG}$ \\
$116 / 60 \mathrm{SN}$ & $6 / 9 \mathrm{SN}$ & $126 / 36 \mathrm{KP}$ & $6 / 24 \mathrm{KP}$ \\
$136 / 60 \mathrm{SN}$ & $6 / 9 \mathrm{SN}$ & $146 / 60 \mathrm{SG}$ & $6 / 18 \mathrm{SG}$ \\
$156 / 18 \mathrm{SN}$ & $6 / 12 \mathrm{SN}$ & $166 / 60 \mathrm{SG}$ & $6 / 60 \mathrm{SG}$ \\
$176 / 60 \mathrm{SN}$ & $6 / 6 \mathrm{SN}$ & $186 / 120 \mathrm{SN}$ & $6 / 120 \mathrm{SN}$ \\
\hline
\end{tabular}

$\overline{\mathrm{SN}}=$ Snellen, $\mathrm{SG}=$ Sheridan - Gardener, $\mathrm{KP}=$ Kay's pictures. 